\title{
Archéopages
}

Archéologie et société

45 | 2017

De la terre au pot

\section{Technique et société. Débat}

Propos recueillis par Catherine Chauveau

\section{Olivier Dargaud, Philippe Husi et Catherine Chauveau}

\section{(2) OpenEdition}

Journals

Édition électronique

URL : https://journals.openedition.org/archeopages/3469

DOI : 10.4000/archeopages.3469

ISSN : 2269-9872

Éditeur

INRAP - Institut national de recherches archéologiques préventives

\section{Édition imprimée}

Date de publication : 1 mars 2018

Pagination : 118-123

ISSN : 1622-8545

\section{Référence électronique}

Olivier Dargaud, Philippe Husi et Catherine Chauveau, «Technique et société. Débat », Archéopages [En ligne], 45 | 2017, mis en ligne le 01 janvier 2020, consulté le 10 juin 2021. URL : http://

journals.openedition.org/archeopages/3469; DOI : https://doi.org/10.4000/archeopages.3469 


\section{Technique et société}

\section{La matière ne se comporte pas forcément d'une manière intuitive. \\ Faire une céramique, cela ne s'improvise pas. Faire des fours, cela s'apprend. S'interroger sur ces savoir-faire mène à se demander de quels phénomènes sociaux ils naissent et lesquels ils génèrent.}

\section{8}

\section{Débat Olivier Dargaud}

chimiste de formation, est adjoint au directeur du département de la création et de la production, en charge de la recherche appliquée, à la Cité de la céramique Sèvres \& Limoges. Pour en savoir plus sur les arts céramiques modernes: A. Brongniart, Traité des arts céramiques ou des poteries, considérées dans leur histoire, leur pratique et leur théorie, Paris, 184.4 La terre transfigurée. 250 ans de porcelaine à Sèvres, Alternatives, coll. Paradoxe Altern, 2017.

Pour visionner Le Souffle de Sèvres, série de films sur les cuissons au four à bois réalisées en 2016 à la manufacture de Sèvres : http://www.sevresciteceramique.fr/ site.php?type $=$ P\&id $=861$

\section{Philippe Husi}

membre du Laboratoire Archéologies et Territoires (LAT) au sein de l'UMR CITERES, pilote le réseau européen de chercheurs consacré à la céramique médiévale et moderne (Iceramm). Il a publié notamment : avec Y. Henigfeld, F. Ravoire et L. Bellanger, «L'approvisionnement des villes médiévales (XII ${ }^{\mathrm{e}}-\mathrm{XVI}^{\mathrm{e}}$ siècles $)$ dans le nord de la France à partir de l'étude de la céramique », in E. Lorans et X. Rodier (dir), Archéologie de l'espace urbain, $137^{\mathrm{e}}$ congrès du comité des travaux historiques et scientifiques, Tours, PUFR/CTHS, coll. Perspectives Villes et Territoires, 2014, p. 419-431; La céramique du haut Moyen Âge dans le centre-ouest de la France. De la chronologie aux aires culturelles, $49^{\mathrm{e}}$ suppl. Revue Archéologique du Centre de la France, Tours, FERAC/ ARCHEA, 2013 ; avec Y. Henigfeld, « La céramique médiévale dans la moitié nord de la France. Bilan et perspectives », in J. Chapelot (dir.), Trente ans d'archéologie médiévale en France. Un bilan pour un avenir, $9^{\mathrm{e}}$ congrès international de la Société d'archéologie médiévale (Vincennes 16/18 juin 2006), Caen, Publications du Craham, 2010, p. 305-320. 





Philippe Husi La céramique est indestructible et donc omniprésente dans les niveaux archéologiques. Son étude nous permet d'aborder quasi tous les aspects de la vie d'une société : la technique, la structure sociale, l'économie, l'esthétique, les différences et les transferts de culture... Et ce sur des millénaires d'histoire humaine, avec une variété de matériaux, de formes et d'usages inouie. La porcelaine est une production tout à fait à part dans ce riche corpus, relativement récente dans nos régions et réservée aux têtes couronnées. À quoi tient cette place particulière, selon vous?

Olivier Dargaud La porcelaine fascine les Européens dès qu'ils la découvrent au Moyen Âge. Elle vient de très loin, coûte très cher et est associée à d'autres produits rares et luxueux. Elle fascine aussi parce qu'elle est très blanche. Dans notre environnement, il n'y a pas tant que cela d'objets totalement blancs. Et le blanc a une valeur symbolique de pureté forte en Occident. Si la porcelaine est bien cuite, son décor ne s'altère pas, et cette notion d'inaltérabilité a un attrait puissant. Et là, on atteint le contraste saisissant entre la pureté, la délicatesse, la fraîcheur de l'objet fini et la brutalité de ses conditions de production : des roches dures, des températures proches de celles du magma... Encore un motif de fascination. Et enfin, la porcelaine entre dans ces possessions que seuls les plus riches des riches peuvent obtenir, et que l'on cherche donc à copier et à produire en quantité. Dès qu'on a su faire cela, son usage s'est largement démocratisé. Pour les générations de la première moitié $\mathrm{du} \mathrm{xx}^{\mathrm{e}}$ siècle, la porcelaine, bien que devenue courante, est réservée aux « grandes occasions »; le service en porcelaine était encore le cadeau de mariage par excellence. Aujourd'hui, la porcelaine peut être produite à bas coût et on en trouve à très faible prix dans n'importe quel supermarché, où elle est, comme les autres formes de céramique, en compétition avec le plastique ; d'ailleurs, ces deux matériaux sont historiquement liés $\mathbf{1}^{\mathbf{1}}$. Mais la céramique a un avantage indéniable : le plastique se détruit, et sinon, se désagrège peu à peu ; la céramique, non. C'est un matériau très ancien qui reste très... contemporain.

PH Et c'est un matériau lié à l'innovation ! Je ferai un parallèle entre la porcelaine et le grès. Le grès

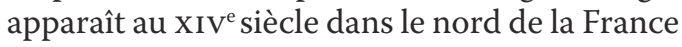
essentiellement, pour le transport des produits alimentaires, et cela grâce à une combinaison de facteurs : la recherche de meilleure conservation, certes, mais en lien avec l'apparition de considérations hygiénistes; la capacité toute récente de monter des fours à haute température, à $1100 / 1300{ }^{\circ} \mathrm{C}$; la volonté d'élargir les zones de diffusion commerciale. L'adoption du grès comme matière d'emballage a permis à certaines régions de trouver des nouveaux marchés : par exemple, la viande des porcs normands et bretons a pu être diffusée sur tout l'ouest de la France. Évidemment, il y a d'abord des phases d'expérimentation de composition de pâte et de techniques de cuisson où l'on a produit ce que l'on appelle du proto-grès, mais la persévérance dans ces recherches est due, selon moi, à des demandes sociales fortes, et par conséquent, sans doute aussi à l'implication d'élites locales. Dans quel contexte est née la production de porcelaine en France?

OD On est fin $\mathrm{XVII}^{\mathrm{e}}$, début $\mathrm{XVIII}^{\mathrm{e}}$, et ce qui va amener une première révolution se passe à Meissen, en Allemagne. Un savant, connu pour chercher à créer la pierre philosophale, va réussir à fabriquer des creusets d'un matériau très résistant en chauffant à très haute température ses matières premières. Selon cette histoire, il fait les premières porcelaines dures « à la manière des Chinois » et le fait savoir. Très vite, les puissances nationales comprennent qu'on a franchi une étape dans l'imitation, jusque-là très imparfaite, des porcelaines importées. Plusieurs manufactures naissent, disparaissent parfois, autour de la fabrication de ces compositions vitrifiées, ou semi-vitrifiées, exemptes de coloration naturelle (exemptes de fer, notamment) ou bien teintées par des pigments blancs (par de l'étain, par exemple). Toutes gardent leurs secrets de fabrication et on sent bien que c'est un enjeu fort de pouvoir et d'argent. Le climat nationaliste montant fait que la France entre dans une compétition avec l'Allemagne et mobilise ses savants. La manufacture de Sèvres apparaît officiellement en 1754, quelques décennies après la manufacture royale de Meissen ${ }^{2}$, et trouver le secret de la "pâte dure », et donc la bonne matière et la bonne façon de la travailler, mettra quasi quinze ans ; ce qui est relativement court et a été possible grâce à la grande effervescence intellectuelle en géologie, en chimie, en sciences globalement, de cette époque. Aux XVIII et $\mathrm{XIX}^{\mathrm{e}}$ siècles, il n'y a pas la séparation entre science et art telle qu'on la connait aujourd'hui. On n'innove pas dans l'un sans innover dans l'autre. Il y a une fusion des mondes qui est bien plus forte, une vision plus globale des problématiques et des enjeux de connaissance. Il s'agit de faire les plus beaux services pour les rois, certes, mais très vite aussi, de tester les limites techniques, d'aller vers la prouesse ; on a, par exemple, du mal à faire tenir debout certains vases tant ils sont grands. C'est une période qui annonce la première révolution industrielle. Puis, une fois cette phase passée, au début du $\mathrm{xx}^{\mathrm{e}}$ siècle, s'opère un retour vers l'art, qui va être le moteur de l'innovation à Sèvres. Â présent, les deux moteurs - technique et artistique - vont de pair : les artistes demandent à Sèvres quelque chose qui ne s'est jamais vu et qui s'appuie sur une technique qui n'a jamais été opérée.

PH Lorsqu'on travaille sur des ateliers de production, on constate qu'ils sont installés à proximité de ressources en eau, en bois et en argile. Les analyses chimiques, pétrographiques, que nous réalisons pour déterminer les provenances des argiles, montrent que, pour les époques médiévales vient de la plastique

la pâte utilisée

par les sculpteurs

2. Fabrique de porcelaine de Saxe fondée en 1710. 


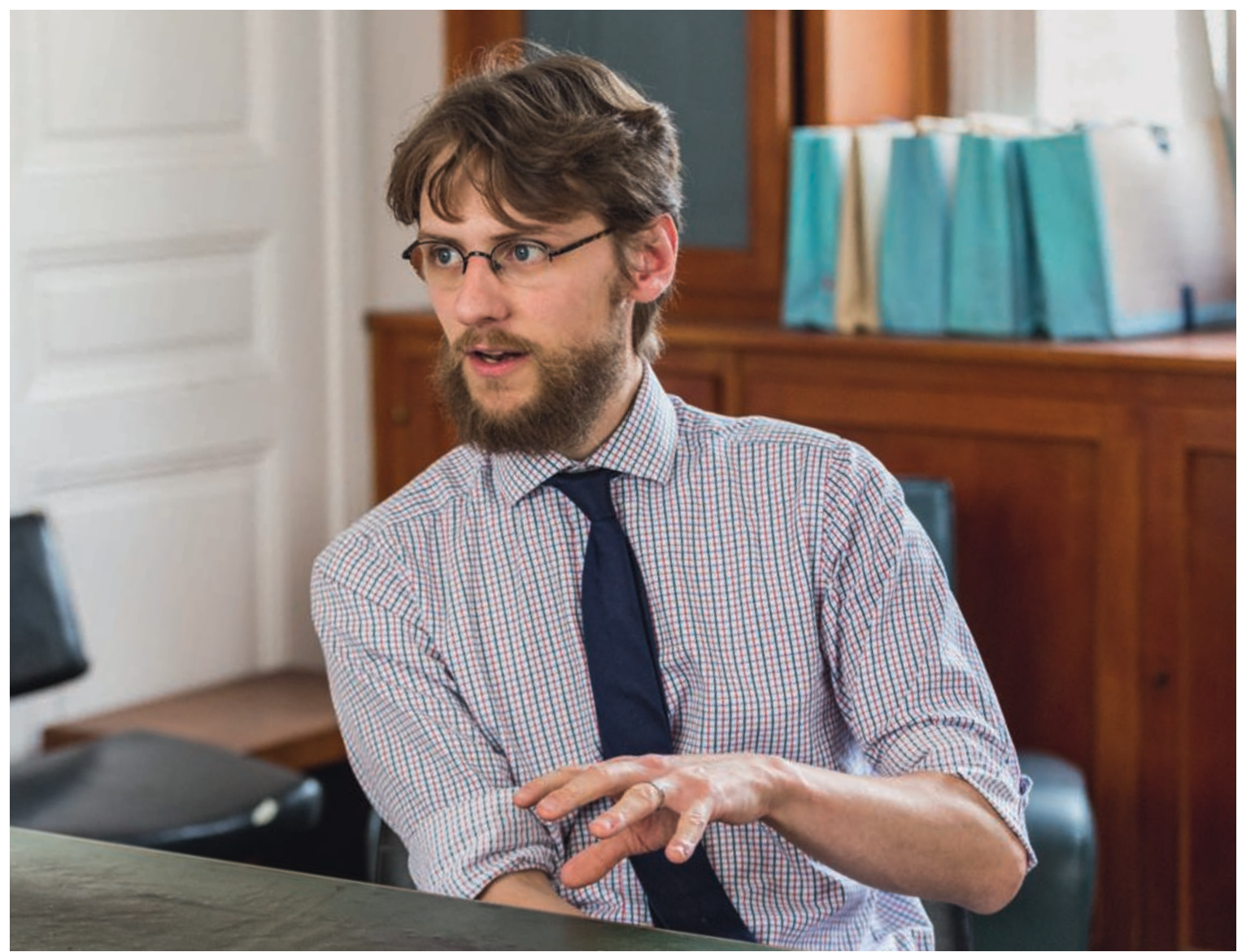

\section{On peut s'interroger sur ce qui fait qu'une société, à l'échelle locale ou nationale, est prête à entretenir une caste de céramistes au service d'une production.}

Olivier Dargaud

et modernes, elles viennent des environs, et ce même pour des ateliers qui ont une grosse production. Ce n'est pas du tout le cas pour Sèvres, mais c'est une question d'échelle, évidemment. OD Oui, si on a une diffusion locale, les potiers vont chercher une bonne terre, mais pas forcément la quantité. Pour la manufacture royale, la volonté est d'emblée une production nationale pour inonder l'Europe, ce qui entraîne un vrai jeu de piste aux gisements les plus intéressants mais une implantation proche du pouvoir et des institutions savantes. Il existe des céramiques kaolinitiques depuis l'Antiquité, considérées déjà comme étant de haute qualité, et plusieurs zones de gisements étaient connues. Il est remarquable qu'on ait compris en Europe que c'était le kaolin qui allait être le composant principal des pâtes ; la pâte dure de Sèvres, elle est à 70 \% de kaolin, c'est l'une des plus riches en kaolin d'Europe, encore aujourd'hui. $\mathrm{Au} \mathrm{XVIII}^{\mathrm{e}}$ siècle, le kaolin extrait en Limousin et en région lyonnaise est apparu comme le plus abondant et d'excellente qualité. L'autre composante majeure est d'arriver à de très hautes températures. On monte à $1400^{\circ} \mathrm{C}$ là où les Chinois, bien qu'ils aient une culture largement millénaire sur ces techniques de haut feu, ne dépassent pas les $1300{ }^{\circ} \mathrm{C}$. Quand on voit la difficulté d'obtenir les derniers 100 degrés, c'est un avantage qui ne va pas sans effort! Comment a-t-on passé ce seuil ? C'est encore un grand questionnement. Dans les textes, c'est parfois attribué au hasard, souvent lié à l'apparition $\mathrm{du}$ four rond dans les environs de Limoges. Cette forme permet d'obtenir ces hautes températures, avec une séparation très nette entre ce qu'on appelle le petit feu et le grand feu. Le début de la cuisson est opéré à la manière habituelle (le petit feu) puis, à un moment donné, on modifie la façon de gérer le feu, on passe au grand feu. De tout cela naît une nouvelle culture professionnelle qui sépare nettement les céramistes grand feu de ceux du petit feu.

PH Mais existe-t-il une forme de transmission entre ces deux cultures ou forment-elles des circuits complètement différents?

OD Ce sont des circuits très séparés avec quand même des liens. Séparés parce que les porcelaines 
étaient réservées au roi, liés parce que, par exemple, ce sont les mêmes émaux qui sont utilisés en faïence et en porcelaine. Il y a forcément un aller et retour qui se fait entre les deux productions. On sait que dans les émaux de Palissy, on a pratiquement les mêmes fusions au plomb que dans les premiers émaux de Sèvres. Et quant à la question de savoir quelle est la bonne taille d'un four par rapport à son chargement, que l'on soit en grand feu ou en petit feu, cela fait partie des discussions sur les formes et tailles de four, incessantes, j'imagine, depuis l'apparition de la poterie. PH Oui, et l'on voit comment des formes disparaissent et réapparaissent, et se modifient au long des siècles. Les fours immenses se trouvent aussi bien dans l'Antiquité, les fours à sigillées, qu'à l'époque moderne, lorsque le but est d'avoir de très grosses productions. On y cuit pendant un à plus de deux jours de nombreuses pièces. Les fours que nous voyons à Sèvres sont très proches des fours à grès de Puisaye. Même lorsqu'il s'agit de vaisselle commune, l'investissement est tel, en bois notamment, qu'on ne peut pas se permettre d'avoir trop de ratés de cuisson. Car, même si tout ce qui est vendable est vendu, une partie de la production était perdue ; la présence de ratés de cuisson est d'ailleurs l'un des indices archéologiques probants de présence d'un atelier de poterie. En ce qui concerne la porcelaine, ces tests à répétition pour atteindre les règles générales de ce qu'est «le » bon four sont très bien exposés dans le livre d'un fabricant de four à Limoges, Jacques Coudamy ${ }^{3}$, qui a travaillé de la fin des fours à bois à l'arrivée des fours à gaz, entre les années 1950 et 1980.

OD Limoges a été le lieu de nombreuses expérimentations : le four à moufles, par exemple, né de l'idée de mutualiser plusieurs moufles pour un seul feu. Cela donne de gigantesques ensembles. Ces réflexions ont ensuite abouti à l'utilisation des fours tunnels où l'on maintient un feu constant ${ }^{4}$ (et donc une température constante) dans le four et c'est la sole portant les pièces à cuire qui avance dans le four. Mais en général, pour la porcelaine, ce sont plutôt de petits fours. On voit bien que l'on se retrouve avec une forme de continuité avec des expériences précédentes comme celle que vous évoquiez pour le grès.

3. Constructeur de fours pour porcelaine à Limoges et auteur de 240 années de porcelaine, qui présente l'histoire des techniques et des hommes de 1771 à 2011 ; en vente au musée du Four des Casseaux à Limoges

(museedescasseaux.com) 4. L'apparition des fours à feu continu date de 1809 .

5. Actuellement, à

Sèvres, on considère qu'il faut entre 5 et 7 ans pour que cette transmission soit accomplie. phénomène de cheminée, qui lui-même entretient le fait qu'on aspire plus d'air, ce qui accroît la dépense de calories à l'intérieur et, du coup, cela alimente le feu de cheminée et ainsi de suite. On ne peut pas avoir une cheminée qui serait trop grande ou trop large par rapport aux tailles des pièces à cuire. Que le four soit rond ou ovale, il doit être voûté car c'est ce qui optimise au maximum le chargement et la cuisson sans dépense de trop de combustible. Ceci dit, ne nous leurrons pas. Cette « apparition » de techniques de fabrication de la porcelaine, on tente de l'expliquer, de chercher des modèles... On écrit une histoire qui se perpétue pleine de pionniers, de secrets, de choses exaltantes, qui laisse dans l'ombre d'autres aspects. Ce n'est pas propre ni confortable de faire de la porcelaine : on a les mains dans la boue, dans l'eau, on a froid aux doigts... Mais cela se sublime, par le feu, d'abord, mais aussi par ce que l'on met de savoir-faire et par la valeur de la pièce produite. Tous ceux qui participent de la production, aussi ingrates que puissent apparaître leurs tâches, vont prendre leur part de cette valeur. Aujourd'hui comme hier, les artisans se trouvent régulièrement dans une situation où on leur demande de faire des choses sans précédent. Ils sont donc perpétuellement dans l'apprentissage de quelque chose d'inédit pour eux, bien que pas forcément inédit au regard de l'histoire. Même si beaucoup de ces innovations sont perdues, l'importance de la passation de maître à apprenti est primordiale et reconnue ${ }^{\mathbf{5}}$. Et la création repose sur cette haute maîtrise technique. Ce facteur social est-il décelable en archéologie?

PH Ce sont des interrogations relativement récentes qui nous sont venues par le biais des études en ethnologie. Les archéologues, les médiévistes, en tout cas, ont longtemps considéré la poterie comme une activité majoritairement domestique, au mieux comme un métier d'appoint. Alors que les ethnologues ont observé, dans toutes les cultures, que la fabrication des poteries est liée à une catégorie de personnes, que les diverses tâches peuvent aussi être réparties selon les positions sociales, l'âge, le sexe etc. Ce métier est le fruit d'un apprentissage issu de traditions et d'innovations. Il y a, parfois, une population entière qui vit de ces artisanats. Dans le milieu de la porcelaine, existe-t-il une hiérarchie des postes et selon quels critères?

OD Aujourd'hui, la séparation des métiers n'a plus vraiment lieu d'être parce que ce sont tous des techniciens des métiers d'art. Mais historiquement, il y a une forme de graduation entre les trois grands métiers : celui qui donne la forme, celui qui donne la couleur et celui qui mène au résultat. Â quel point c'était marqué socialement, je ne sais pas. Je pense qu'à Sèvres ce qui est particulier, c'est que l'outil de production est dès le départ au service de l'art et au service de la science dans un échange renouvelé avec des institutions scientifiques et avec des artistes. C'est cela, je pense, qui a 


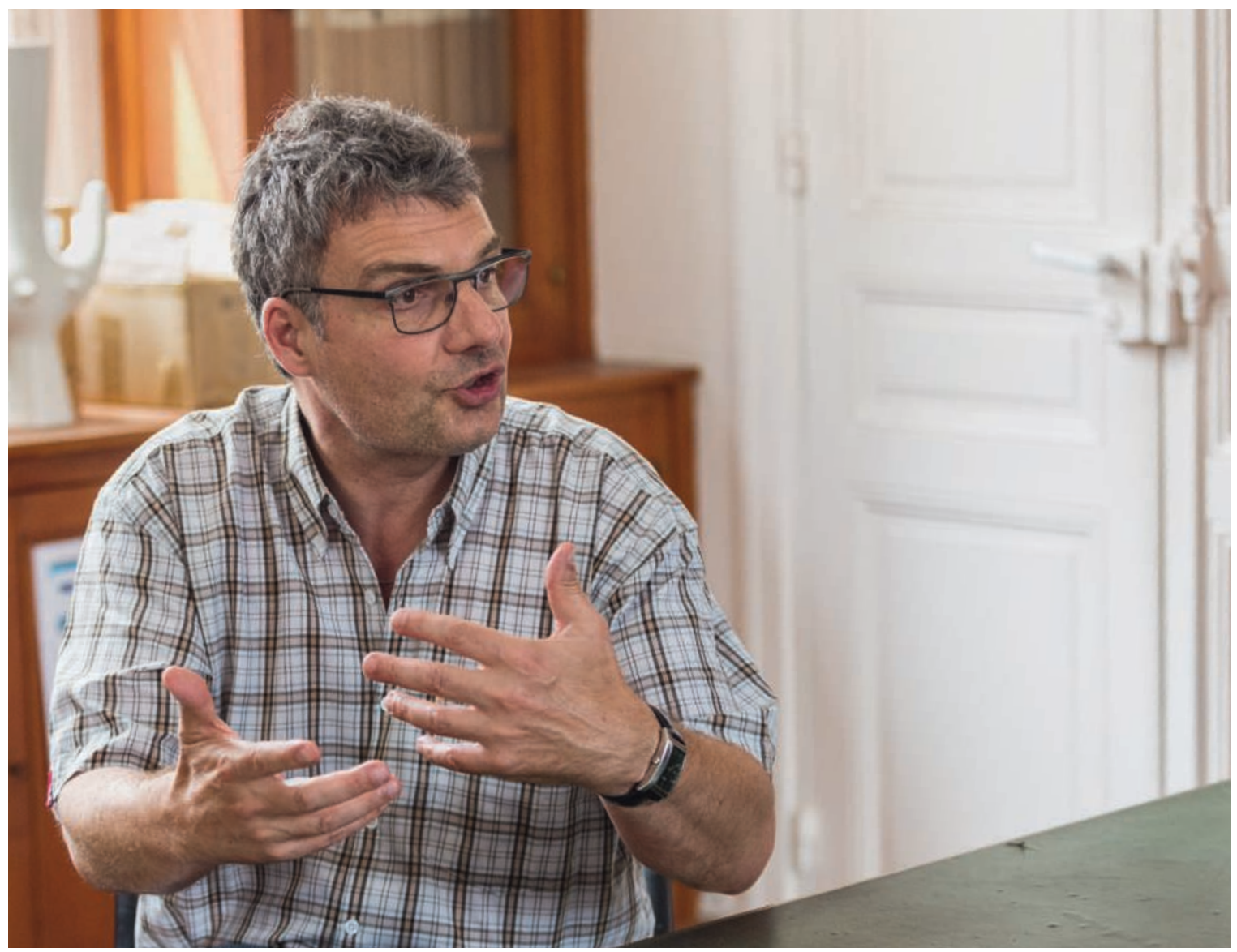

\section{$\mathrm{Ne}$ parlons pas uniquement d'invention car l'innovation technique est aussi faite de résurgences et d'adaptations.}

Philippe Husi

maintenu la portée nationale de la manufacture. Alors que dans un bassin de production, le contexte est plus circonscrit même s'il peut y avoir des fulgurances créatrices. Quel type d'échanges pouvaient avoir les potiers médiévaux?

PH Les échanges entre potiers existent. Ils sont bien documentés pour l'Antiquité, pour la production de sigillée notamment. Mais je dirais que la proximité géographique d'artisans différents a été un autre facteur majeur de l'innovation. À partir de l'époque carolingienne, quand la plupart des villes prennent de l'essor, on constate que les artisans se regroupent et entretiennent des relations. Est-ce une coïncidence, c'est alors qu'on voit surgir, par exemple, la céramique glaçurée. Et ce, un peu partout en Europe du nordouest sans qu'un foyer d'émergence détermine sa diffusion. En revanche, on constate que potiers et artisans du métal travaillent souvent aux mêmes endroits, avec, peut-être, un accès au plomb plus aisé pour les premiers conjugué à une demande de vaisselle plus luxueuse pour une élite urbaine. Il est vrai que ce type de céramique glaçurée existe en Italie auparavant. Mais il semble qu'il n'y ait pas eu de transfert technologique entre ces lieux de production italiens et ceux du nord-ouest de la France. Ce qui, par contre, a été le cas pour la faïence, la meilleure preuve étant chronologique, avec une quasi contemporanéïté des découvertes au sud comme au nord de l'Europe. Venue d'Italie et d'Espagne, cette production gagne, relativement lentement, le sud puis le nord de la France : à Marseille et à Lyon, au XVI ${ }^{\mathrm{e}}$ siècle ; en Touraine, pas avant le milieu du XVII ${ }^{\mathrm{e}}$ siècle. Et finalement, les faïenceries sont présentes partout au XVIII ${ }^{\mathrm{e}}$ siècle, grâce à une conjonction de facteurs : imitation, transmission, effet de mode, mobilité des artisans... Et nous travaillons sur la compréhension de ces phénomènes dans la longue durée. Qu'est-ce qui suscite l'émergence d'un type de céramique, son développement, sa longévité ?

OD C'est toute l'histoire de la manufacture de Sèvres! Un lieu qui fait vivre et crée son histoire chaque jour, avec pour mission de la consigner et de la transmettre, et toujours dans la dynamique de l'histoire de l'art et de l'histoire des sciences. Que l'on s'interroge sur ses racines lointaines, comme sur ses racines finalement assez récentes, on a besoin de comprendre d'où l'on vient. 